\title{
Resveratrol pretreatment attenuates injury and promotes proliferation of neural stem cells following oxygen-glucose deprivation/reoxygenation by upregulating the expression of $\mathrm{Nrf} 2, \mathrm{HO}-1$ and $\mathrm{NQO} 1$ in vitro
}

\author{
CHANGBO SHEN, WEI CHENG, PINGPING YU, LI WANG, LULIN ZHOU, LI ZENG and QIN YANG
}

\begin{abstract}
Department of Neurology, The First Affiliated Hospital of Chongqing Medical University, Chongqing 400016, P.R. China
\end{abstract}
Received July 13, 2015; Accepted July 18, 2016

DOI: $10.3892 / \mathrm{mmr} .2016 .5670$

\begin{abstract}
There is considerable interest in the use of drugs and other methods for protecting implanted neural stem cells (NSCs) from the adverse environment of injured tissue for successful cell therapy. Resveratrol can modify cardiac stem cells to enhance their survival and differentiation, however, its effect and the mechanism underlying its neuroprotective effect on NSCs following stroke remain to be fully elucidated. Nuclear factor erythroid 2-related factor 2 ( $\mathrm{Nrf}-2$ ) signaling is important in antioxidative stress, and the role of Nrf-2 signaling in the enhanced neuroprotection of NSCs by resveratrol following stroke also remains to be elucidated. In the present study, NSCs were pretreated with resveratrol prior to oxygen-glucose deprivation/reoxygenation (OGD/R) in vitro. The survival, apoptosis and proliferation of the NSCs were assessed using an MTT assay, Hoechst 33258 staining of nuclei and flow cytometry, respectively. In addition, the activity of superoxide dismutase (SOD), level of malondiadehyde (MDA) and content of glutathione (GSH) were determined. The protein expressions levels of Nrf-2, NAD(P)H:quinone oxidoreductase 1 (NQO-1), and heme oxygenase 1 (HO-1) were detected using western blot analysis. It was found that resveratrol markedly enhanced NSC survival and proliferation, decreased apoptosis and the levels of MDA, and increased the activity of SOD and content of GSH in a concentration-dependent manner following OGD/R injury in vitro. In addition, the protein expression levels of Nrf2, HO-1 and NQO1 were significantly upregulated. These findings suggested that resveratrol attenuated injury and promoted proliferation of the NSCs, at least in part, by upregulating the expression of Nrf2, HO-1 and NQO1 following OGD/R injury in vitro.
\end{abstract}

Correspondence to: Professor Qin Yang, Department of Neurology, The First Affiliated Hospital of Chongqing Medical University, 1 Youyi Road, Yuzhong, Chongqing 400016, P.R. China E-mail: xyqh200@126.com

Key words: resveratrol, oxygen-glucose deprivation/reoxygenation, neural stem cells, oxidative stress, apoptosis

\section{Introduction}

Neural stem cell (NSC) implant therapy offers considerable potential for the regeneration or self-repair of injury or degeneration of the brain or spinal cord. However, the transplantation of exogenous NSCs is limited due to low survival rates and differentiation of the implanted NSCs. The leading cause of implanted cell death is associated with the microenvironment of the injured tissue, including inflammatory responses, oxidative stress and the development of pro-apoptotic factors. Several attempts have aimed to enhance stem cell survival, proliferation and differentiation following cell therapy (1-3). For example, neurotrophin-3, released from poly( $\varepsilon$-caprolac tone)-block-poly(l-lactic acid-co-e-caprolactone scaffolds, has been shown to promote the survival and neuronal differentiation of transplanted NSCs in a rat spinal cord injury model (4). Hydrogel scaffolding also increases the survival of engrafted NSCs (5). Therefore, there is substantial interest in the identification of drugs and other methods for the protection of implanted cells from the adverse environment of the injured tissue for successful cell therapy.

Resveratrol (trans-3,5,4'-trihydroxystilebene), a naturally occurring polyphenolic phytoalexin, is predominantly found in dietary sources, including grapes, red wine, peanuts, plums and other plants (6). Studies have shown that resveratrol has anti-inflammatory, anti-oxidant, anticancer and chemopreventive properties $(7,8)$. In addition, it has been reported that resveratrol has neuroprotective effects in ischemic cerebral stroke, Alzheimer disease and Parkinson's disease (9-11). Resveratrol can decrease neuronal apoptosis by upregulating the expression of B cell lymphoma-2 (Bcl-2) and downregulating the expression of $\mathrm{Bcl}-2$-associated $\mathrm{X}$ protein in the hippocampus following focal cerebral ischemia in rats (12). Resveratrol also enhances hippocampal neurogenesis and alleviates hippocampal atrophy in prenatally stressed rats and mice with chronic fatigue $(13,14)$. Previously, it was observed that resveratrol pretreatment can attenuate cerebral ischemic injury by upregulating the expression levels of the nuclear factor erythroid 2-related factor 2 (Nrf-2) and heme oxygenase 1 (HO-1) transcription factors to improve neurological function (15). Gorbunov et al (16) also found that resveratrol can modify cardiac stem cells to enhance their survival and 
differentiation, and to improve cardiac function. However, whether resveratrol can decrease injury and promote proliferation of NSCs following cerebral ischemic damage remain to be fully elucidated.

Nrf2, a cap'n'Collar (CNC) transcription factor, is important in antioxidative stress (17). When cells or organisms are exposed to oxidative stress, electrophiles or chemopreventive agents, the Nrf2 pathway is activated to trigger the expression of antioxidant response element (ARE)-dependent genes, including HO-1 and NAD(P) $\mathrm{H}$ :quinone oxidoreductase 1 (NQO1), which attenuate cellular oxidative stress $(18,19)$.

Therefore, the present study investigated whether and how resveratrol pretreatment decreases injury and promotes the proliferation of NSCs following oxygen-glucose deprivation/reoxygenation (OGD/R) in vitro. The results showed that resveratrol markedly and dose-dependently enhanced NSC survival and proliferation, decreased NSC apoptosis and the levels of malondiadehyde (MDA), increased SOD activity and glutathione (GSH) content, and upregulated the protein expression levels of Nrf2, HO-1 and NQO1 following OGD/R injury of NSCS in vitro.

\section{Materials and methods}

NSC culture. The NSCs were obtained from eight neonatal 1-2 day-old male $(n=5)$ and female $(n=3)$ Sprague-Dawley rats, provided by the Department of Animal Experiments, Chongqing Medical University (Chongqing, China), and used for primary NSC cultivation, as described previously with modifications $(20-22)$. The rats were maintained at $20 \pm 2^{\circ} \mathrm{C}$, humidity $60 \pm 5 \%$ under a 12 -h light/dark cycle with access to food and water ad libitum. The rats were sacrificed by immersion in $75 \%$ ethanol for $5 \mathrm{~min}$ prior to cervical dislocation. The cerebellum was dissected from the brains. Briefly, the cerebral cortices were dissected, washed with Dulbecco's modified Eagle's medium/F12 (DMEM/F12) medium (Gibco; Thermo Fisher Scientific, Inc., Waltham, MA, USA) and digested with $0.125 \%$ trypsin at $37^{\circ} \mathrm{C}$ for $30 \mathrm{~min}$. The tissue was then dissociated into a single cell suspension by mechanical titration with a sterile, fire-polished glass Pasteur pipette. The cells were counted and suspended at a density of $5 \times 10^{5} / \mathrm{ml}$ in a $25-\mathrm{cm}^{2}$ glass culture flask in DMEM/F12 medium supplemented with 2\% B27 (Gibco; Thermo Fisher Scientific, Inc.), $20 \mathrm{ng} / \mathrm{ml}$ recombinant human epidermal growth factor, $20 \mathrm{ng} / \mathrm{ml}$ recombinant human basic fibroblast growth factor (Peprotech, Inc., Rocky Hill, NJ, USA), $100 \mathrm{mg} / \mathrm{ml}$ streptomycin and $100 \mathrm{U} / \mathrm{ml}$ penicillin (GE Healthcare Life Sciences Hyclone Laboratories), Logan, UT, USA). The cells were maintained at $37^{\circ} \mathrm{C}$ in an incubator with a humidified atmosphere of $5 \%$ carbon dioxide, and half the medium was replaced every other day. The cells were passaged approximately every 7 days. When passaging, the neurospheres were incubated in $0.125 \%$ trypsin for $5 \mathrm{~min}$ and dissociated mechanically into a single-cell suspension using a sterile, fire-polished glass Pasteur pipette. Following centrifugation at $79 \mathrm{x}$ g for $5 \mathrm{~min}$ at room temperature, the cells were reseeded in new glass culture flasks at the same density as in the primary culture. The third passage NSCs were inoculated at a density of $5 \times 10^{5} / \mathrm{ml}$ into plastic culture flasks coated with poly-d-lysine (Beyotime Institute of Biotechnology, Jiangsu, China) and laminin (Sigma-Aldrich, St. Louis, MO, USA) for adherent culture.

All experimental procedures were performed with the approval of the Animal Experimental Committee of Chongqing Medical University, and submitted to relevant laws.

OGD/R model. According to previously described methods, OGD/R of the cortical NSCs was performed to mimic cerebral artery occlusion and reperfusion injury $(23,24)$. Briefly, following washing twice with D-Hanks solution (GE Healthcare Life Sciences Hyclone Laboratories), The NSCs were incubated with D-Hanks solution at $37^{\circ} \mathrm{C}$ for $150 \mathrm{~min}$ in an incubator with a humidified atmosphere containing $95 \% \mathrm{~N}_{2}$ and $5 \% \mathrm{CO}_{2}$ (Thermo 3111; Thermo Fisher Scientific, Inc.). When reoxygenated, the D-Hanks solution was replaced with NSC medium, and the NSCs were maintained in a humidified normoxic atmosphere containing $5 \% \mathrm{CO}_{2}$ for $24 \mathrm{~h}$ at $37^{\circ} \mathrm{C}$.

Drug treatment. To determine whether resveratrol enhanced NSC viability following OGD/R injury in vitro, four treatment groups were used for comparison: i) Normal group, NSCs were cultured in NSC culture medium without OGD; ii) model group, NSCs were treated with OGD only; iii) ethanol group, NSCs were treated with NSC medium containing ethanol (volume fraction 1.3\%) for $24 \mathrm{~h}$ prior to OGD; iv) resveratrol pretreatment groups, NSCs were maintained in complete medium containing different concentrations (1, 5, 10, 20, 50 and $100 \mu \mathrm{mol} / \mathrm{l}$ ) of resveratrol (purity 99\%; Sigma-Aldrich) for $24 \mathrm{~h}$ prior to $\mathrm{OGD} / \mathrm{R}$. The optimal effect was observed at a concentration of $5 \mu \mathrm{mol} / 1$ resveratrol, and thus 1,5 and $20 \mu \mathrm{mol} / 1$ resveratrol were used for the subsequent experiments.

To investigate whether resveratrol promoted NSC proliferation, and decreased NSC apoptosis and anti-oxidative stress, three treatment groups were used for comparison: i) Normal group, NSCs were cultured in NSC culture medium without OGD; ii) model group, NSCs were treated with OGD only; iii) resveratrol pretreatment groups, NSCs were maintained in complete medium containing different concentrations $(1,5$ and $20 \mu \mathrm{mol} / \mathrm{l}$ ) of resveratrol for $24 \mathrm{~h}$ prior to OGD/R.

To examine the effects of resveratrol on the protein expression levels of Nrf-2, NQO-1 and HO-1 in the NSCs following OGD/R, three groups were used for comparison: i) Normal group, NSCs were cultured in NSC culture medium without OGD; ii) model group, NSCs were treated with OGD only; iii) $5 \mu \mathrm{mol} / 1$ resveratrol pretreatment group, NSCs were maintained in complete medium containing $5 \mu \mathrm{mol} / 1$ resveratrol for $24 \mathrm{~h}$ prior to $\mathrm{OGD} / \mathrm{R}$.

MTT cell viability assay. The MTT assay (Beyotime Institute of Biotechnology) was used to quantitatively measured cell viability. Briefly, the third passage NSCs $(\sim 5,000$ cells/well $)$ were seeded into poly-L-lysine-coated 96 -well plates, with six replicates in each group, and subjected to the various treatments described above. Following treatment, MTT solution $(5 \mathrm{mg} / \mathrm{ml})$ was added to each culture well, and the NSCs were incubated for $3 \mathrm{~h}$ at $37^{\circ} \mathrm{C}$. The medium was carefully removed, and the blue formazan products were dissolved with $200 \mu$ l DMSO per well. The absorbance was then measured on a microplate 
reader (Thermo Labsystems, Vantaa, Finland) at $570 \mathrm{~nm}$, with cell survival rates expressed as a percentage of absorbance value of cells without any treatment. Each experiment was repeated three times.

Flow cytometric analysis of NSC proliferation. Cell cycle analysis was performed using a BD FACS Vantage SE flow cytometer (BD Biosciences, Franklin Lakes, NJ, USA). The NSCs in the different culture conditions were harvested synchronously, dissociated into single cells, resuspended in $70 \%$ ethanol and stored at $4^{\circ} \mathrm{C}$ overnight. The cells were then washed with PBS, centrifuged at $124 \mathrm{x}$ g for $5 \mathrm{~min}$ at room temperature and resuspended in $0.5 \mu \mathrm{l}$ buffer containing propidium iodide (50 $\mu \mathrm{g} / \mathrm{ml}$; Sigma-Aldrich) and RNase (100 $\mu \mathrm{g} / \mathrm{ml}$; Sigma-Aldrich) for $30 \mathrm{~min}$. Flow cytometry was then performed to detect cell cycle. Each experiment was repeated three times.

Measurements of SOD activity, MDA levels and GSH content. To measure the activity of SOD, the NSCs were lysed with RIPA lysis buffer and centrifuged at $124 \mathrm{x} \mathrm{g}$ for $5 \mathrm{~min}$ at room temperature, according to the manufacturer's protocol at $4^{\circ} \mathrm{C}$ (Beyotime Institute of Biotechnology). This method uses xanthine and xanthine oxidase to generate superoxide radicals, which react with p-iodonitrotetrazolium violet to form a red formazan dye. The absorbances of each standard and sample were read at $450 \mathrm{~nm}$ using a 96-well microplate reader, and the background absorbance from culture medium, which was not used for any cell cultures, was determined. SOD activity was expressed as U/mg protein. Each experiment was repeated three times.

The levels of MDA were measured using the thiobarbituric acid method. Briefly, the NSCs were harvested and homogenized. An Enhanced BCA Protein Assay kit (Beyotime Institute of Biotechnology) was used to determined the quantity of total protein in the supernatant of the NSC homogenate. The absorbance was measured at $532 \mathrm{~nm}$ by spectrometry. Each experiment was repeated three times.

For the GSH assay, the cells were lysed with RIPA lysis buffer and the cell suspension was centrifuged at $124 \mathrm{x} \mathrm{g}$ for $5 \mathrm{~min}$ at room temperature, according to the manufacturer's protocol, at $4^{\circ} \mathrm{C}$ (Beyotime Institute of Biotechnology), and the supernatant was used for further analyses. The content of GSH was measured on the principle that reduced GSH can be quantified by colorimetry at $405 \mathrm{~nm}$ when it reacts with 5,5'-Dithiobis (2-nitrobenzoic acid). The absorbance of GSH was measured at $405 \mathrm{~nm}$. Cellular GSH content was calculated using a concurrently run GSH standard curve and expressed as nmol of GSH per milligram of cellular protein $(\mathrm{nmol} / \mathrm{mg}$ protein). Each experiment was repeated three times.

Hoechst 33258 staining of nuclei for analysis of apoptotic cells. Hoechst 33258 staining of nuclei was used to detect apoptotic cells. Hoechst 33258 , a blue fluorescent dye, readily permeates the cell membrane for the determination and semi-quantification of cells with fragmented and condensed chromatin. Following the $24 \mathrm{~h}$ re-oxygenation period, the NSCs were washed three times with PBS and fixed in $4 \%$ formaldehyde for $10 \mathrm{~min}$. The fixed cells were then washed with PBS and incubated with Hoechst 33258 (Sigma-Aldrich) for $5 \mathrm{~min}$ in a humidified chamber, protected from light, at $37^{\circ} \mathrm{C}$. The cells were washed three times with PBS and mounted with one drop of mounting solution. The cell nuclei were observed and images were captured using a fluorescence microscope (Olympus BX51; Olympus Corporation, Tokyo, Japan).

Western blot analysis. Following the various treatments described above, the cellular proteins were extracted and the protein levels were analyzed. Briefly, the cells were rinsed with ice-cold PBS and lysed using a nuclear and cytoplasmic protein extraction kit (Beyotime Institute of Biotechnology), according to the manufacturer's protocol. The concentrations of nuclear and cytoplasmic protein were measured using a BCA protein assay kit. The sample lysates $(50 \mu \mathrm{g})$ were loaded onto a 10\% SDS-PAGE gel for electrophoresis, following which the protein was transferred onto a $0.45 \mu \mathrm{m}$ PVDF membrane (EMD Millipore, Billerica, MA, USA), blocked in 5\% skim milk powder-TBST buffer, and incubated with primary antibodies and horseradish peroxidase (HRP)-conjugated secondary antibodies. The membranes were incubated with the appropriate primary antibodies at $4{ }^{\circ} \mathrm{C}$ overnight. The primary antibodies used were as follows: Polyclonal rabbit anti-Nrf-2 (1:1,000; cat. no. ab62352), polyclonal goat anti-NQO-1 (1:1,000; cat. no. ab2346), monoclonal rabbit anti-HO-1 (1:1,000; cat. no. ab68477) and anti- $\beta$-actin $(1: 1,000$; cat. no. ab8229) all obtained from Abcam (Hong Kong, China). The membranes were then treated with HRP-conjugated goat anti-rabbit (cat. no. ZDR-5306) and rabbit anti-goat (cat. no. ZDR-5308) secondary antibodies (1:100; Beijing Zhongshan Golden Bridge Biotechnology Co. Ltd., Beijing, China) for $1 \mathrm{~h}$ at $37^{\circ} \mathrm{C}$. The antigen-antibody complexes were then detected with an ECL reagent kit (Beyotime Institute of Biotechnology), and visualized and analyzed using the ChemiDoc ${ }^{\mathrm{TM}} \mathrm{XRS}+$ imaging system (Bio-Rad Laboratories, Inc., Hercules, CA, USA). The results were quantified using QuantityOne 1-D analysis software (Bio-Rad Laboratories, Inc.). The quantities of each product were normalized by dividing the average gray level of the signal by that of the corresponding $\beta$-actin amplicon. Each experiment was repeated three times.

Cell counts. The numbers of positively-stained cells were counted in 10 randomly-selected visual fields (magnification, x200) for each sample using a BX51 fluorescence microscope and analysis using Image-Pro Plus 6.0 (Media Cybernetics, Inc., Rockville, MD, USA).

Statistical analysis. Statistical analysis was performed using the SPSS 19.0 statistical software package (IBM SPSS, Armonk, NY, USA). All values are expressed as the mean \pm standard deviation. Every experiment was repeated at least three times. Data were statistically analyzed by one-way analysis of variance and Tukey's post-hoc test. $\mathrm{P}<0.05$ were considered to indicate a statistically significant difference.

\section{Results}

Resveratrol pretreatment decreases injury and promotes proliferation of NSCs in a concentration-dependent manner following OGD/R in vitro. An MTT assay was used to determine the optimal concentration of resveratrol pretreatment on NSC viability following OGD/R in vitro. As shown in Fig. 1, 


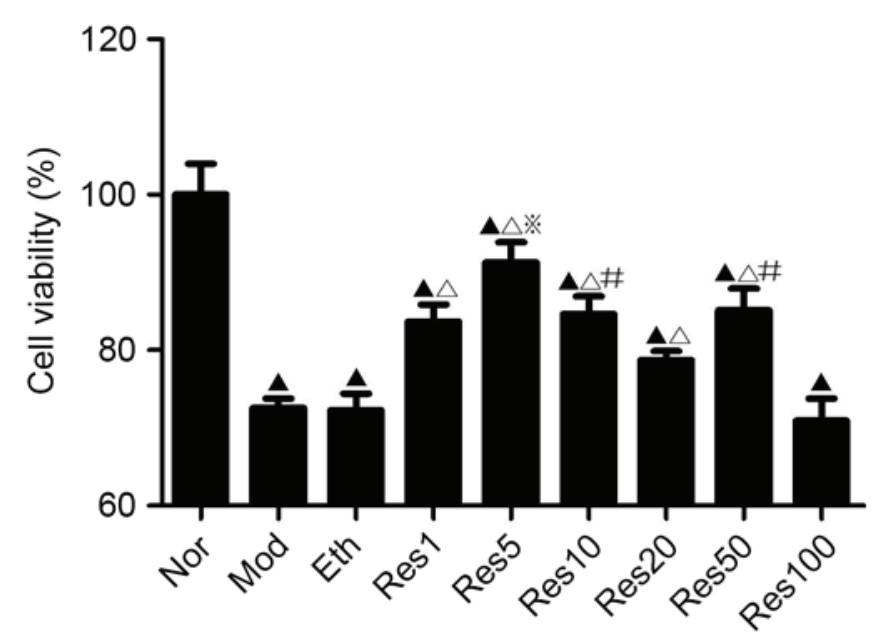

Figure 1. Resveratrol promotes NSC viability in a concentration-dependent manner following OGD/R injury. The MTT assay revealed that NSC viability was significantly reduced in the Mod, Eth and Res groups, compared with the Nor group following OGD/R. No significant differences was found between the Mod, Eth and $100 \mu \mathrm{mol} / 1$ resveratrol (Res100) groups. However, NSC viability was significantly higher in the $1,5,10$ and $50 \mu \mathrm{mol} / 1$ resveratrol (Res1, 5, 10 and 50) groups, compared with the Mod group, with the highest viability in the $5 \mu \mathrm{mol} / 1$ resveratrol (Res5) group. ${ }^{\wedge} \mathrm{P}<0.05$, vs. Nor group; ${ }^{\Delta} \mathrm{P}<0.05$, vs. Mod group; ${ }^{*} \mathrm{P}<0.05$, vs. Res1, Res10, Res20, Res50 and Res100 groups; " $\mathrm{P}<0.05$, vs. Res 20 group. Data are presented as the mean \pm standard deviation and were compared using one-way analysis of variance $(n=3$ for each group). NSC, neural stem cell; OGD/R, oxygen-glucose deprivation/reoxygenation; Nor, normal; Mod, model; Eth, ethanol; Res, resveratrol.

NSC viability was significantly reduced in the model, ethanol and resveratrol groups compared with the normal group. No differences were found between the model, ethanol and $100 \mu \mathrm{mol} / 1$ resveratrol groups. However, NSC viability in the resveratrol groups $(1,5,10$ and $50 \mu \mathrm{mol} / \mathrm{l})$ was significantly higher, compared with that of the model group, and the highest viability was observed in the $5 \mu \mathrm{mol} / 1$ resveratrol group. These results showed that resveratrol decreased the OGD/R-induced injury of the NSCs and the most effective concentration of resveratrol was $5 \mu \mathrm{mol} / 1$. Therefore, 1,5 and $20 \mu \mathrm{mol} / 1$ resveratrol were selected for further experiments.

To investigate whether resveratrol decreases the apoptosis of NSCs following OGD/R injury in vitro, apoptotic NSCs were labeled with Hoechst 33258, a blue fluorochrome, which penetrates the cell membrane. Hoechst 33258-positive cells under the fluorescence microscope were indicative of apoptotic cells. As shown in Fig. 2A, few Hoechst 33258-positive cells were observed in the normal group; there were significantly higher numbers in the control and resveratrol groups (Fig.2B-F). The numbers of Hoechst 33258-positive cells in the resveratrol groups were significantly lower, compared with that in the control group, and were lowest in the $5 \mu \mathrm{mol} / 1$ resveratrol group. These results showed that resveratrol decreased the apoptosis of NSCs following OGD/R injury in vitro.

To investigate whether resveratrol can promote the proliferation of NSCs following OGD/R injury in vitro, proliferated NSCs were examined using flow cytometry. As shown in Fig. 3, the ratio of NSCs in the S and G2/M phase was significantly higher in the normal group (Fig. 3A), compared with the model group (Fig. 3B), and in the resveratrol groups, compared with the model group, and was highest in the $5 \mu \mathrm{mol} / 1$ resvera- trol group (Fig. 3C-F). These result suggested that resveratrol promoted proliferation of the NSCs following OGD/R in vitro.

Taken together, the above results showed that resveratrol decreased injury and promoted the proliferation of NSCs in a concentration-dependent manner following OGD/R injury in vitro. Furthermore, the most effective concentration of resveratrol was $5 \mu \mathrm{mol} / 1$.

Resveratrol pretreatment attenuates oxidative damage and upregulates protein expression levels of $\mathrm{Nrf}-2, \mathrm{NQO}-1$ and HO- 1 in NSCs following OGD/R injury in vitro. Oxidative stress was assessed by evaluating the products of lipid peroxidation with the levels of MDA, enzymatic activity of SOD and content of GSH. As shown in Fig. 4A, the levels of MDA in the control and resveratrol groups were significantly higher, compared with that in the normal group. However, the levels of MDA in the resveratrol groups were significantly lower, compared with that in the control group, and was lowest in the $5 \mu \mathrm{mol} / 1$ resveratrol group (Fig. 4A). The activity of SOD and content of GSH were significantly lower in the control group, compared with the normal group $(\mathrm{P}<0.05)$, and were significantly higher in the resveratrol pretreatment groups, compared with the normal and control groups, with the highest levels observed in the $5 \mu \mathrm{mol} / 1$ resveratrol groups (Fig. 4B and C). These results indicated that resveratrol pretreatment ameliorated oxidative damage of the NSCs following OGD/R in vitro.

$\mathrm{Nrf} 2$, a CNC transcription factor, regulates the expression of numerous reactive oxygen species (ROS), detoxifying agents and antioxidants. The components of the Nrf2 signaling pathway in mammals include Nrf2, NQO-1 and HO-1. Therefore, the present study examined whether resveratrol upregulated the expression levels of Nrf2, NQO-1 and HO-1.

Western blot analysis (Fig. 5A) showed that the protein expression levels of Nrf2 in the nuclei, and NQO-1 and HO-1 in the cytoplasm of NSCs were significantly upregulated in the model and resveratrol groups, compared with those in the normal group. The highest levels were observed in the $5 \mu \mathrm{mol} / 1$ resveratrol group $(\mathrm{P}<0.05$; Fig. 5B-D). These results indicated that resveratrol pretreatment upregulated the protein expression levels of Nrf2, NQO-1 and HO-1 of the NSCs following OGD/R in vitro.

\section{Discussion}

The present study showed that resveratrol pretreatment increased the viability, ameliorated the apoptosis and promoted the proliferation of NSCs following OGD/R injury in vitro. This occurred in a concentration-dependent manner, and the most effective concentration of resveratrol was $5 \mu \mathrm{mol} / \mathrm{l}$. An increasing number of reports have shown that resveratrol has a general range of activities, which depend on several factors, including the concentration of resveratrol, the cell or organism type being examined, and the physiological or pathological state of the cells or organisms. Moriya et al (14) reported that resveratrol $(40 \mathrm{mg} / \mathrm{kg}$ body weight) promoted hippocampal neurogenesis and ameliorated hippocampal atrophy in mice with chronic fatigue. Harada et al (25) found that resveratrol enhanced hippo- 
A

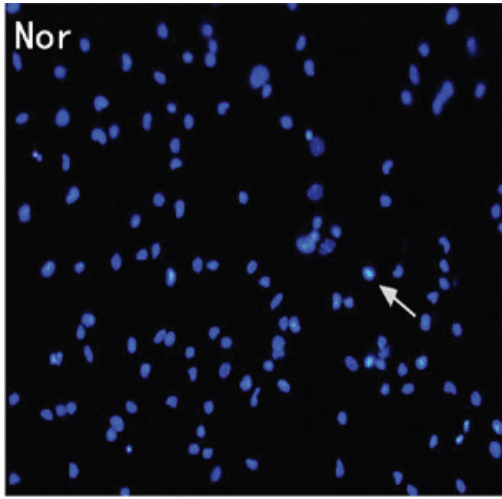

C

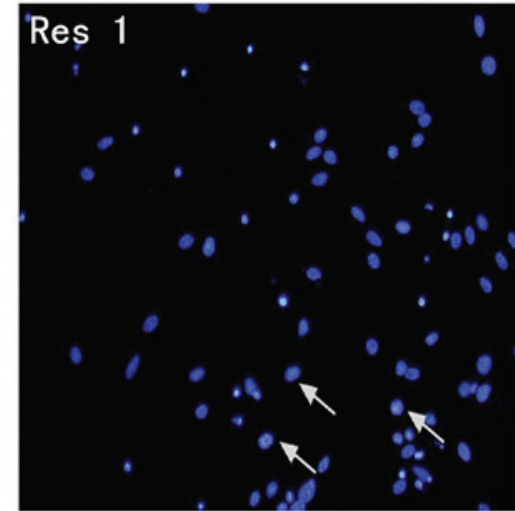

$\mathbf{E}$

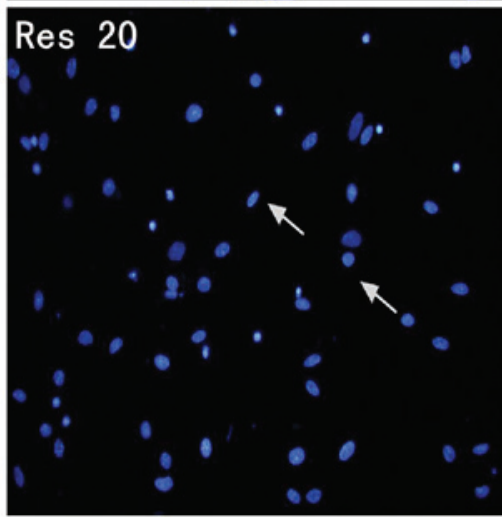

B

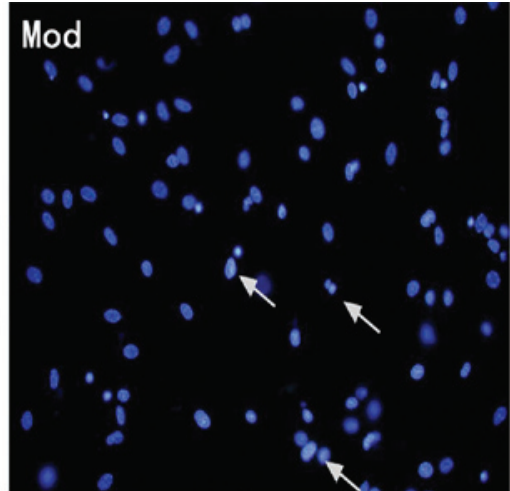

D

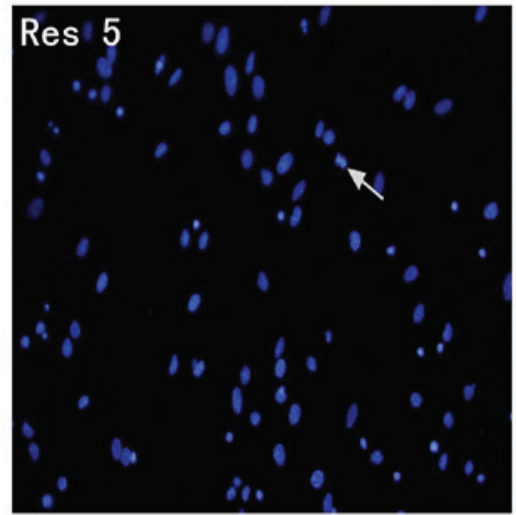

$\mathbf{F}$

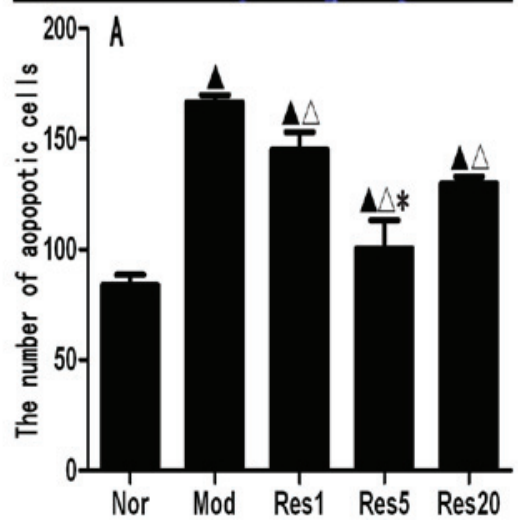

Figure 2. Resveratrol decreases apoptosis of NSCs in a concentration-dependent manner following OGD/R injury. Apoptosis was determined using Hoechst 33258 staining (magnification, x400). Few Hoechst 33258-positive cells were identified in the (A) Nor group, whereas there were significantly higher numbers in the (B) Mod and the (C-E) Res1, 5 and 20 groups. The numbers of Hoechst 33258-positive cells in the Res groups were significantly lower, compared with that in the Mod group, and were lowest in the $5 \mu \mathrm{mol} / 1$ resveratrol (Res5) group. (F) Graph showing the numbers of Hoechst 33258-positive NSCs in each group. These results suggested that Res reduced apoptosis of the NSCs following OGD/R injury in vitro. $\Delta \mathrm{P}<0.05$, vs. Nor group; $\triangle \mathrm{P}<0.05$, vs. Mod group; ${ }^{*} \mathrm{P}<0.05$, vs. Res1 and Res 20 groups. Data are presented as the mean \pm standard deviation and were compared using one-way analysis of variance ( $\mathrm{n}=3$ for each group). NSCs, neural stem cells; OGD/R, oxygen-glucose deprivation/reoxygenation; Nor, normal; Mod, model; Res, resveratrol.

campal neurogenesis and improved cognitive function in wild-type mice. Madhyastha et al (13) also reported that resveratrol enhanced postnatal hippocampal neurogenesis in prenatally stressed rats. However, Park et al (26) reported that resveratrol inhibited the proliferation of neural progenitor cells in culture (20 and $50 \mu \mathrm{mol} / \mathrm{l})$ and hippocampal neurogenesis (1-10 $\mathrm{mg} / \mathrm{kg}$ body weight). Leong et al (27) also found that $30-120 \mu \mathrm{mol} / 1$ resveratrol inhibited the proliferation of embryonic cardiomyoblast. In the present study, it was observed that $1-50 \mu \mathrm{mol} / 1$ resveratrol promoted NSC viability and $100 \mu \mathrm{mol} / 1$ resveratrol decreased NSC viability following OGD/R injury in vitro. Therefore, further investigations are required to determine whether resveratrol has a dual concentration effect on neurogenesis in different physiological or pathological states.
Oxidative stress generated by ROS is one of the important pathological mechanisms of OGD/R injury (28-30). Following OGD/R injury, excess ROS are generated. As NSCs have low levels of antioxidative enzyme activity, they are vulnerable to ROS, which causes oxidative damage and leads to cell death. Therefore, antioxidant treatment is important in OGD/R injury.

MDA, a product of lipid peroxidation, is an important marker for oxidative stress. In addition, all cells or organisms have defense mechanisms, which involve GSH reductase, SOD, catalase and GSH peroxidase, to protect cells against the damaging effects of ROS (31). SOD and GSH, two important endogenous free radical scavengers, are crucial in maintaining the oxidative and antioxidative balances of the body, as they scavenge free radicals to protect cells from oxidative damage. Increased activity of SOD and GSH increases 
A

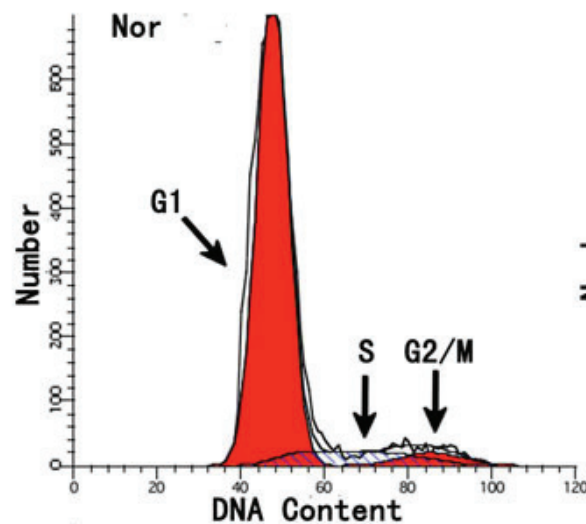

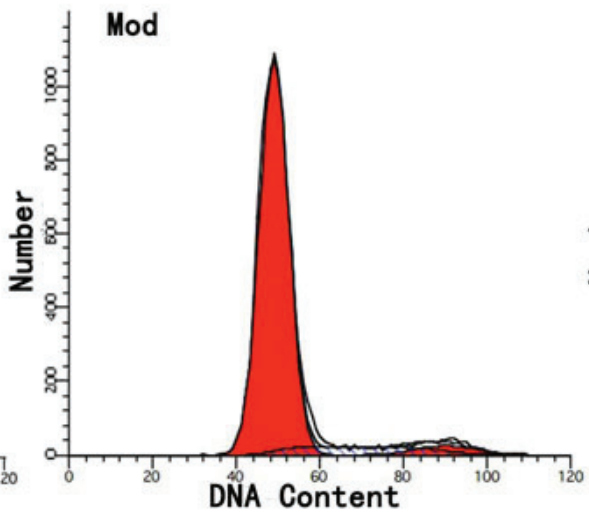

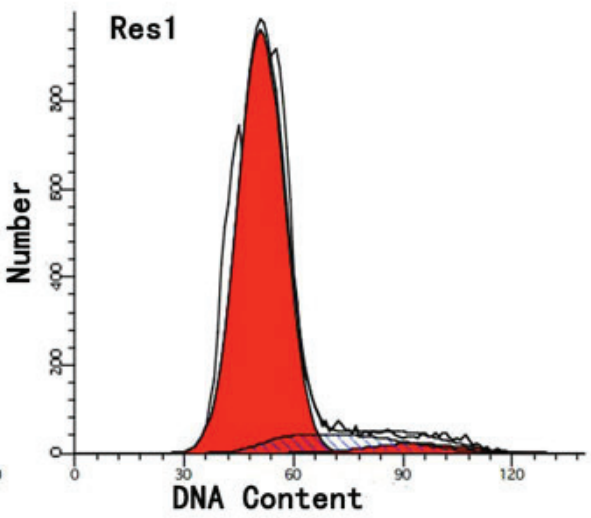

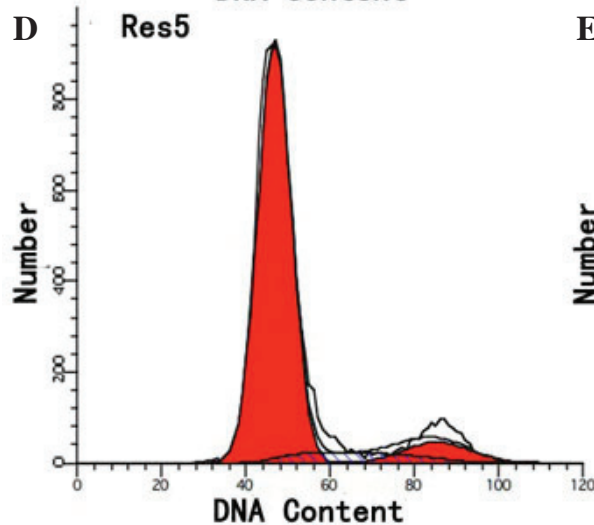
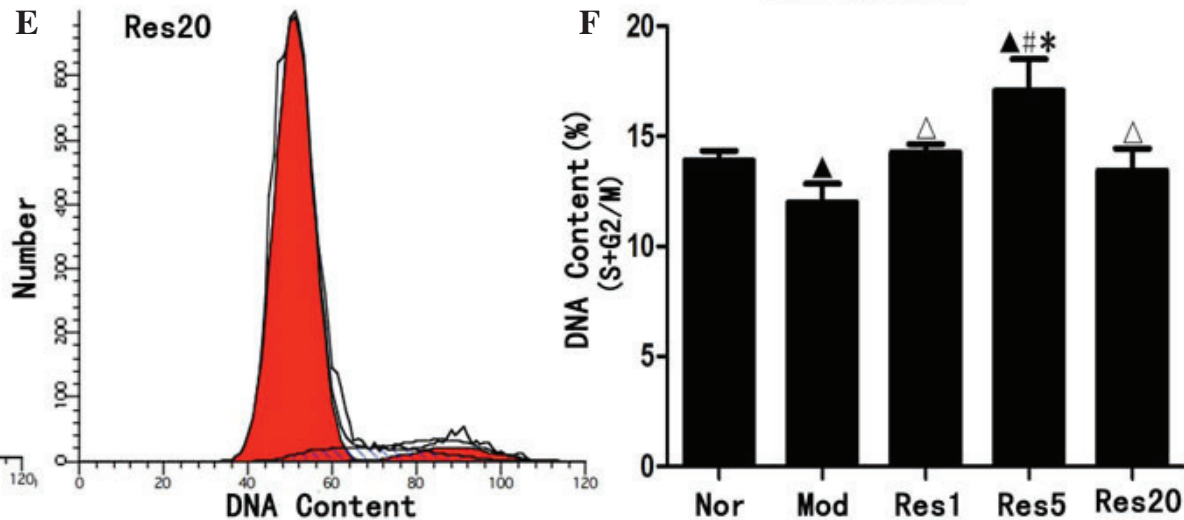

Figure 3. Resveratrol promotes the proliferation of NSCs in a concentration-dependent manner following oxygen-glucose deprivation/reoxygenation injury. Flow cytometry revealed that, compared with the (A) Nor group, the ratio of NSCs in the S and G2/M phase were significantly decreased in the (B) Mod group and, compared with the Mod group, increased in the (C-E) Res groups and, compared with the (C and E) Res1 and Res20 groups, increased in the (D) Res5 group, peaking in the $5 \mu \mathrm{mol} / 1$ (Res5) group. ${ }^{\wedge} \mathrm{P}<0.05$, vs. Nor group; ${ }^{\wedge} \mathrm{P}<0.05$ and ${ }^{*} \mathrm{P}<0.01$, vs. Mod group; ${ }^{*} \mathrm{P}<0.05$, vs. Res1 and Res 20 groups. Data are presented as the mean \pm standard deviation and were compared using one-way analysis of variance ( $\mathrm{n}=3$ for each group). NSCs, neural stem cells; Nor, normal; Mod, model; Res, resveratrol.

\section{A}
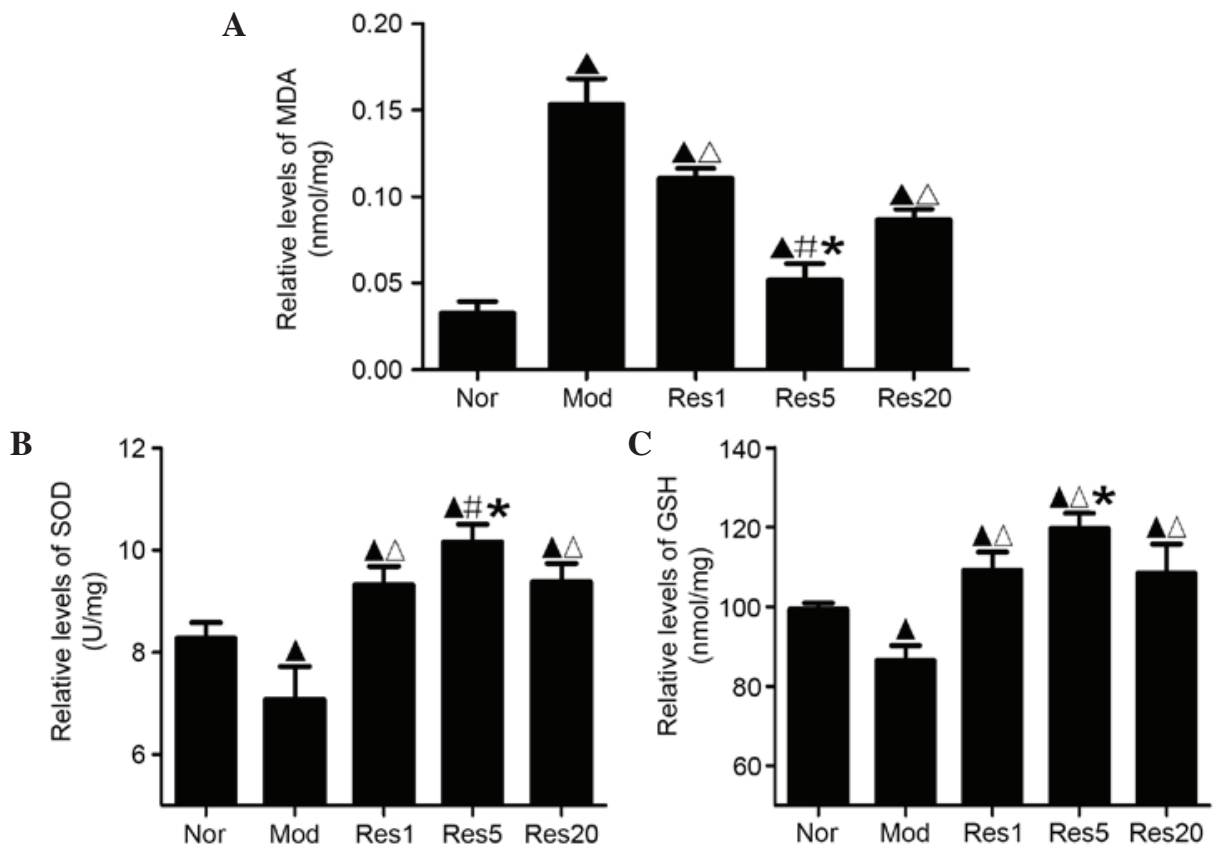

Figure 4. Resveratrol ameliorates oxidative damage of neural stem cells in a concentration-dependent manner following oxygen-glucose deprivation/reoxygenation in vitro. (A) Levels of MDA were significantly higher in the Mod group, compared with the Nor group, and significantly lower in the Res groups, compared with the Mod group, and was lowest in the $5 \mu \mathrm{mol} / 1$ resveratrol (Res5) group. (B) SOD activity and (C) GSH content were significantly lower in the Mod group, compared with the Nor group, and significantly higher in the Res groups, compared with the Nor and Mod groups, being highest in the $5 \mu \mathrm{mol} / 1$ resveratrol (Res5) group. ${ }^{\Delta} \mathrm{P}<0.05$, vs. Nor group; ${ }^{\circ} \mathrm{P}<0.05$, vs. Mod group; ${ }^{~} \mathrm{P}<0.01$ and ${ }^{*} \mathrm{P}<0.05$, vs. Res 1 and Res 20 groups Data are presented as the mean \pm standard deviation and were compared using one-way analysis of variance ( $\mathrm{n}=3$ for each group). Nor, normal; Mod, model; Res, resveratrol; MDA, malondiadehyde; SOD, superoxide dismutase; GSH, glutathione. 
A

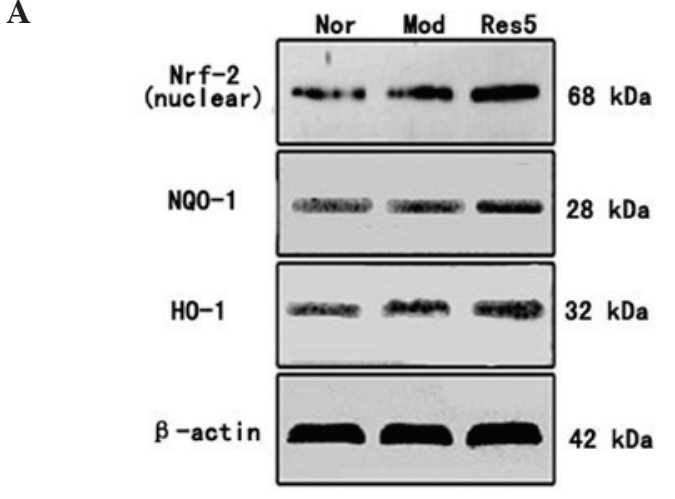

C

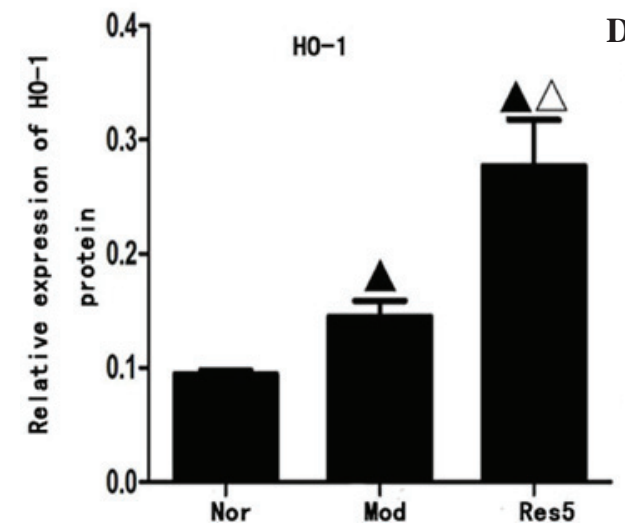

B
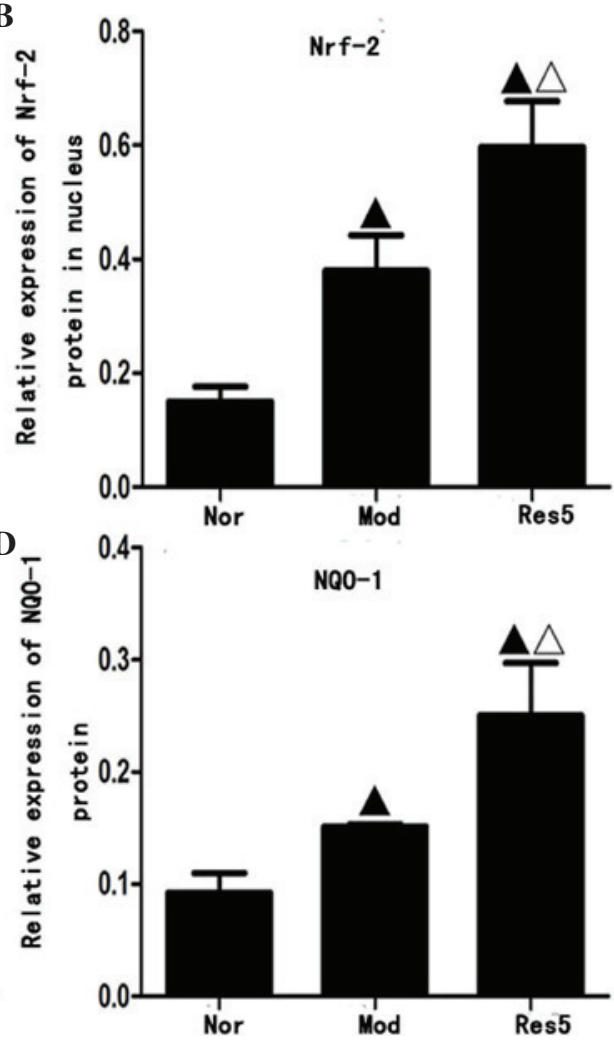

Figure 5. Resveratrol upregulates the protein expression levels of Nrf-2 in the nuclei, and NQO-1 and HO-1 in the cytoplasm of neural stem cells following oxygen-glucose deprivation/reoxygenation injury in vitro. (A) Protein expression of Nrf2 in nuclei and NQO-1 and HO-1 in cytoplasm. Quantification of data for (B) Nrf2, (C) NQO-1 and (D) HO-1. Western blot analysis revealed that the protein expression levels of Nrf2 in the nuclei, and NQO-1 and HO-1 in the cytoplasm were significantly upregulated in the Mod and Res groups, compared with those in the Nor group. The levels were highest in the $5 \mu \mathrm{mol} / 1 \mathrm{resveratrol}$ (Res5) group suggesting resveratrol increased expression of Nrf2 in the nuclei and NQO-1 and HO-1 in the cytoplasm. ${ }^{\mathbf{\Delta}} \mathrm{P}<0.05$, vs. Nor group; ${ }^{\circ} \mathrm{P}<0.05$, vs. Mod group. Data are presented as the mean \pm standard deviation and were compared using one-way analysis of variance ( $\mathrm{n}=3$ for each group). Nor, normal; Mod, model; Res, resveratrol; Nfr-2, nuclear factor erythroid 2-related factor 2; NQO-1 NAD(P)H:quinone oxidoreductase 1; HO-1, heme oxygenase 1.

the ability of cells to eliminate oxygen free radicals. In the present study, the levels of MDA were significantly higher in the control group, compared with the normal group. The results suggested that OGD/R-induced injury of the NSCs involves oxidative stress. Following resveratrol pretreatment, the levels of MDA were significantly decreased. By contrast, the activity of SOD and content of GSH were increased significantly in the resveratrol pretreatment groups, compared with the normal and control groups. Konyalioglu et al (32) reported that resveratrol protects embryonic neural stem cells against hydrogen peroxide-induced oxidative stress (32). Taken together, these findings suggested that resveratrol pretreatment attenuated oxidative damage, and had neuroprotective and neurogenetic effects on the NSCs following $\mathrm{OGD} / \mathrm{R}$ injury in vitro. However, the exact protective mechanisms underlying the effect of resveratrol on NSCs remains to be fully elucidated.

Nrf2 is vital in antioxidative stress $(30,33)$. Under basal conditions, Nrf2-dependent transcription is repressed by a negative regulator, Kelch-like ECH-associated protein 1 (Keap1). When the cells are exposed to oxidative stress, electrophiles or chemopreventive agents, Nrf2 evades Keap1-mediated repression and activates the expression of antioxidant response element (ARE)-dependent genes, including HO-1 and NQO1, to maintain cellular redox homeostasis and attenuate cellular oxidative stress $(18,19,34)$.
Yang et al (35) reported that the expression levels of Nrf2 and HO-1 were upregulated at $3 \mathrm{~h}$ and peaked $24 \mathrm{~h}$ following cerebral ischemia, prior to gradually decreasing at 48 and $72 \mathrm{~h}$ (35). In our previous study, the expression levels of Nrf2 and HO-1 were upregulated at $2 \mathrm{~h}$ and peaked $24 \mathrm{~h}$ following cerebral I/R in rats (15). The present study showed that the protein expression levels of Nrf2 in the nuclei, and NQO-1 and HO-1 in the cytoplasm of the NSCs were significantly dysregulated following OGD/R injury in vitro. These findings suggested that the Nrf2/ARE signaling pathway may be important in vivo and in vitro following stroke or OGD/R injury.

Sakata et al (36) reported that resveratrol upregulates the expression of HO-1 in a time- and dose-dependent manner, and protects against free-radical or excitotoxic damage of cortical neuronal cells in mice in vitro. Resveratrol also protects dopaminergic SH-SY5Y cells against rotenone-induced neurotoxicity by inducing HO-1-dependent autophagy, and upregulating the expression levels of Nrf-2 and NQO-1, which suppresses the oxidative and inflammatory stress responses of a high-fat, high-carbohydrate meal $(37,38)$. In addition, Gorbunov et al (16) and Gurusamy et al (39) reported that resveratrol pretreatment upregulated the expression of $\mathrm{Nrf}-2$, and enhanced implanted multi-potent clonogenic cardiac stem cell survival and proliferation. Our previous study demonstrated that resveratrol pretreatment for 7 days significantly 
decreased cerebral ischemic injury, improved neurological function, upregulated the expression levels of the Nrf2 and HO-1 transcription factors, and ameliorated oxidative damage in rats with middle cerebral artery occlusion (15). In addition, the present study demonstrated that resveratrol pretreatment significantly upregulated the expression levels of Nrf2, NQO-1 and HO-1, decreased injury and promoted the proliferation of NSCs in a concentration-dependent manner following $\mathrm{OGD} / \mathrm{R}$ in vitro. Therefore, these findings suggested that the Nrf2/ARE signaling pathway is vital in the neuroprotection by resveratrol of NSCs against OGD/R injury.

Taken together, the present study demonstrated that resveratrol pretreatment had a neuroprotective effect on NSCs following OGD/R injury. This neuroprotective effect was likely caused, at least in part, by upregulated expression levels of Nrf2, HO-1 and NQO1 to decrease oxidative damage, ameliorate apoptosis and promote neurogenesis. The findings of the present study are important for understanding the mechanism underlying the resveratrol-induced decreased injury and increased proliferation of NSCs. In the future, investigations are required to determine whether Nrf2 signaling mediates resveratrol-induced neurorestorative processes, including neurogenesis, axonal remodeling and oligodendrogenesis, following stroke in vivo.

\section{Acknowledgements}

The present study was supported by grants from the National Key Clinical Specialties Construction Program of China for Neurology [The First Affiliated Hospital of Chongqing Medical University; grant no. (2014)27] and the National Natural Science Foundation of China (grant no. 81071119).

\section{References}

1. Keuters MH, Aswendt M, Tennstaedt A, Wiedermann D Pikhovych A, Rotthues S, Fink GR, Schroeter M, Hoehn M and Rueger MA: Transcranial direct current stimulation promotes the mobility of engrafted NSCs in the rat brain. NMR Biomed 28: 231-239, 2015.

2. Lu P, Graham L, Wang Y, Wu D and Tuszynski M: Promotion of survival and differentiation of neural stem cells with fibrin and growth factor cocktails after severe spinal cord injury. J Vis Exp: e50641, 2014.

3. Sarnowska A, Jablonska A, Jurga M, Dainiak M, Strojek L, Drela K, Wright K, Tripathi A, Kumar A, Jungvid H, et al: Encapsulation of mesenchymal stem cells by bioscaffolds protects cell survival and attenuates neuroinflammatory reaction in injured brain tissue after transplantation. Cell Transplant 22 (Suppl 1): S67-S82, 2013.

4. Tang S, Liao X, Shi B, Qu Y, Huang Z, Lin Q, Guo X and Pei F: The effects of controlled release of neurotrophin-3 from PCLA scaffolds on the survival and neuronal differentiation of transplanted neural stem cells in a rat spinal cord injury model. PLoS One 9: e107517, 2014.

5. Liang Y, Walczak P and Bulte JW: The survival of engrafted neural stem cells within hyaluronic acid hydrogels. Biomaterials 34 : 5521-5529, 2013.

6. Walle T: Bioavailability of resveratrol. Ann N Y Acad Sci 1215: 9-15, 2011.

7. Baur JA and Sinclair DA: Therapeutic potential of resveratrol: The in vivo evidence. Nat Rev Drug Discov 5: 493-506, 2006.

8. Lin HY, Tang HY, Davis FB and Davis PJ: Resveratrol and apoptosis. Ann N Y Acad Sci 1215: 79-88, 2011.

9. Sinha K, Chaudhary G and Gupta YK: Protective effect of resveratrol against oxidative stress in middle cerebral artery occlusion model of stroke in rats. Life Sci 71: 655-665, 2002.
10. Jin F, Wu Q, Lu YF, Gong QH and Shi JS: Neuroprotective effect of resveratrol on 6-OHDA-induced Parkinson's disease in rats. Eur J Pharmacol 600: 78-82, 2008.

11. Li F, Gong Q, Dong H and Shi J: Resveratrol, a neuroprotective supplement for Alzheimer's disease. Curr Pharm Des 18: 27-33, 2012.

12. Li Z, Pang L, Fang F, Zhang G, Zhang J, Xie M and Wang L: Resveratrol attenuates brain damage in a rat model of focal cerebral ischemia via up-regulation of hippocampal Bcl-2 Brain Res 1450: 116-124, 2012.

13. Madhyastha S, Sekhar S and Rao G: Resveratrol improves postnatal hippocampal neurogenesis and brain derived neurotrophic factor in prenatally stressed rats. Int J Dev Neurosci 31: 580-585, 2013.

14. Moriya J, Chen R, Yamakawa J, Sasaki K, Ishigaki Y and Takahashi T: Resveratrol improves hippocampal atrophy in chronic fatigue mice by enhancing neurogenesis and inhibiting apoptosis of granular cells. Biol Pharm Bull 34: 354-359, 2011.

15. Ren JW, Fan C, Chen N, Huang J and Yang Q: Resveratrol pretreatment attenuates cerebral ischemic injury by upregulating expression of transcription factor $\mathrm{Nrf} 2$ and $\mathrm{HO}-1$ in rats. Neurochem Res 36: 2352-2362, 2011.

16. Gorbunov N, Petrovski G, Gurusamy N, Ray D, Kim doH and Das DK: Regeneration of infarcted myocardium with resveratrol-modified cardiac stem cells. J Cell Mol Med 16: 174-184, 2012.

17. Mohagheghi F, Khalaj L, Ahmadiani A and Rahmani B: Gemfibrozil pretreatment affecting antioxidant defense system and inflammatory, but not Nrf-2 signaling pathways resulted in female neuroprotection and male neurotoxicity in the rat models of global cerebral ischemia-reperfusion. Neurotox Res 23: 225-237, 2013.

18. Zhang DD: Mechanistic studies of the Nrf2-Keap1 signaling pathway. Drug metab Rev 38: 769-789, 2006.

19. Ping Z, Liu W, Kang Z, Cai J, Wang Q, Cheng N, Wang S, Wang S, Zhang JH and Sun X: Sulforaphane protects brains against hypoxic-ischemic injury through induction of Nrf2-dependent phase 2 enzyme. Brain Res 1343: 178-185, 2010.

20. Reynolds BA, Tetzlaff W and Weiss S: A multipotent EGF-responsive striatal embryonic progenitor cell produces neurons and astrocytes. J Neurosci 12: 4565-4574, 1992.

21. Andersen RK, Johansen M, Blaabjerg M, Zimmer J and Meyer M: Neural tissue-spheres: A microexplant culture method for propagation of precursor cells from the rat forebrain subventricular zone. J Neurosci Methods 165: 55-63, 2007.

22. Babu H, Claasen JH, Kannan S, Rünker AE, Palmer T and Kempermann G: A protocol for isolation and enriched monolayer cultivation of neural precursor cells from mouse dentate gyrus. Front Neurosci 5: 89, 2011.

23. Chen T, Liu W, Chao X, Qu Y, Zhang L, Luo P, Xie K, Huo J and Fei Z: Neuroprotective effect of osthole against oxygen and glucose deprivation in rat cortical neurons: Involvement of mitogen-activated protein kinase pathway. Neuroscience 183: 203-211, 2011.

24. Wu X, Zhao J, Yu S, Chen Y, Wu J and Zhao Y: Sulforaphane protects primary cultures of cortical neurons against injury induced by oxygen-glucose deprivation/reoxygenation via anti-apoptosis. Neurosci Bull 28: 509-516, 2012.

25. Harada N, Zhao J, Kurihara H, Nakagata N and Okajima K: Resveratrol improves cognitive function in mice by increasing production of insulin-like growth factor-I in the hippocampus. J Nutr Biochem 22: 1150-1159, 2011.

26. Park HR, Kong KH, Yu BP, Mattson MP and Lee J: Resveratrol inhibits the proliferation of neural progenitor cells and hippocampal neurogenesis. J Biol Chem 287: 42588-42600, 2012.

27. Leong CW, Wong CH, Lao SC, Leong EC, Lao IF, Law PT, Fung KP, Tsang KS, Waye MM, Tsui SK, et al: Effect of resveratrol on proliferation and differentiation of embryonic cardiomyoblasts. Biochem Biophys Res Commun 360: 173-180, 2007.

28. Slemmer JE, Shacka JJ, Sweeney MI and Weber JT: Antioxidants and free radical scavengers for the treatment of stroke, traumatic brain injury and aging. Curr Med Chem 15: 404-414, 2008.

29. Nassar NN, Abdelsalam RM, Abdel-Rahman AA and Abdallah DM: Possible involvement of oxidative stress and inflammatory mediators in the protective effects of the early preconditioning window against transient global ischemia in rats. Neurochem Res 37: 614-621, 2012. 
30. Oh YI, Kim JH and Kang CW: Protective effect of short-term treatment with parathyroid hormone 1-34 on oxidative stress is involved in insulin-like growth factor-I and nuclear factor erythroid 2-related factor 2 in rat bone marrow derived mesenchymal stem cells. Regul Pept 189: 1-10, 2014.

31. De la Lastra CA and Villegas I: Resveratrol as an antioxidant and pro-oxidant agent: Mechanisms and clinical implications. Biochem Soc Trans 35: 1156-1160, 2007.

32. Konyalioglu S, Armagan G, Yalcin A, Atalayin C and Dagci T: Effects of resveratrol on hydrogen peroxide-induced oxidative stress in embryonic neural stem cells. Neural Regen Res 8: 485-495, 2013.

33. Maher J and Yamamoto M: The rise of antioxidant signaling-the evolution and hormetic actions of Nrf2. Toxicol App Pharmacol 244: 4-15, 2010.

34. Quincozes-Santos A, Bobermin LD, Latini A, Wajner M, Souza DO, Gonçalves CA and Gottfried C: Resveratrol protects C6 astrocyte cell line against hydrogen peroxide-induced oxidative stress through heme oxygenase 1. PloS One 8: e64372, 2013.
35. Yang C, Zhang X, Fan $\mathrm{H}$ and Liu Y: Curcumin upregulates transcription factor Nrf2, HO-1 expression and protects rat brains against focal ischemia. Brain Res 1282: 133-141, 2009.

36. Sakata Y,Zhuang H,Kwansa H, Koehler RC and Doré S: Resveratrol protects against experimental stroke: Putative neuroprotective role of heme oxygenase 1. Exp Neurol 224: 325-329, 2010.

37. Lin TK, Chen SD, Chuang YC, Lin HY, Huang CR, Chuang JH, Wang PW, Huang ST, Tiao MM, Chen JB and Liou CW: Resveratrol partially prevents rotenone-induced neurotoxicity in dopaminergic SH-SY5Y cells through induction of heme oxygenase-1 dependent autophagy. Int J Mol Sci 15: 1625-1646, 2014.

38. Ghanim H, Sia CL, Korzeniewski K, Lohano T, Abuaysheh S, Marumganti A, Chaudhuri A and Dandona P: A resveratrol and polyphenol preparation suppresses oxidative and inflammatory stress response to a high-fat, high-carbohydrate meal. J Clin Endocrinol Metab 96: 1409-1414, 2011.

39. Gurusamy N, Ray D, Lekli I and Das DK: Red wine antioxidant resveratrol-modified cardiac stem cells regenerate infarcted myocardium. J Cell Mol Med 14: 2235-2239, 2010. 